\title{
No detection of c-kit gene mutations in exons 9, 11, 13 and 17 and low CD117 expression in plaque-stage mycosis fungoides*
}

\author{
David Aldo De Luca ${ }^{1}$, Paula Andrea Enz ${ }^{1}$, Ricardo Luis Galimberti ${ }^{1}$, Adriana Raquel Rinflerch ${ }^{1}$
}

DOI: http:/ /dx.doi.org/10.1590/abd1806-4841.20187930

\begin{abstract}
The growth factor receptor c-kit (CD117) is expressed in immature T-cells and in some advanced forms of mycosis fungoides. c-kit gene mutation results in unrestricted neoplastic proliferation. We aimed to detect by PCR the most frequent exon mutations in seventeen plaque-stage MF patients, in their perilesional skin and in healthy skin donors. We secondarily evaluated CD117 expression by immunohistochemistry in plaque-stage and tumor-stage MF. We detected no mutation in c-kit gene and low CD117 expression was confirmed on atypical cells in one patient. Complete c-kit exon and intron sequences should be assessed and more sensitive sequencing method could be also applied.
\end{abstract}

Keywords: Mycosis fungoides; Proto-oncogene proteins c-kit; Stem cell factor

Mycosis fungoides (MF) is the most common type of primary cutaneous T-cell lymphoma (CTCL). The underlying molecular events are poorly understood; however, genomic and chromosomal instability are common features in CTCL. Classification of early (ES-MF) and advanced MF depends on clinical and histologic criteria, along with blood involvement and metastatic disease. Firstline therapies for ES-MF are topical steroids, psoralen plus UVA or narrowband UVB phototherapy. The prognosis of MF may worsen when atypical T-cells develop a more malignant phenotype under an immunosuppressive microenvironment. ${ }^{1-3}$

CD117 or c-kit is a growth factor receptor with tyrosine kinase activity embedded in the plasma membrane. The c-kit gene is located on chromosome 4q12 and includes 21 exons. C-KIT participates in skin pigmentation, survival of bone marrow stem cells, T-cells and mast cells development, spermatogenesis, steroidogenesis, angiogenesis and electrical pacemaker in the gastrointestinal tract. c-kit gene sequence variations were found in gastrointestinal stromal tumors (GIST), mucosal and acral lentiginous melanoma, mastocytosis, germ cell tumors and adult acute myeloid leukemia. In particular, mutations in exons 9, 11, 13 and 17 activate c-kit constitutively, leading to an unrestricted tumor cell proliferation. Although Brauns et al. described CD117 expression in 27\% MF, 50\% MF with transformation to a large T-cell lymphoma and 60\% Sézary syndrome, so far there are no publications related to c-kit gene mutations in MF. ${ }^{4-6}$

The purpose of this study was to assess the presence of c-kit gene mutations in exons 9, 11, 13 and 17 in plaque-stage ES-MF skin, and in apparently normal perilesional skin and healthy skin samples as control. Our secondary objective was to assess the expression of CD117 by immunohistochemistry (IHC) on those plaque-stage ES-MF patients.

We conducted a prospective case-control study at "Hospital Italiano de Buenos Aires", Argentina. Our local ethics committee approved both the study protocol (number 2199, act 6, page 10,

\footnotetext{
Received 04 December 2017. Accepted 14 May 2018.

* Work conducted at the Dermatology Department, Hospital Italiano de Buenos Aires, Buenos Aires, Argentina.

Financial support: Department of Dermatology and "Instituto de Ciencias Básicas y Medicina Experimental", Hospital Italiano de Buenos Aires. Conflict of interest: None.

1 Dermatology Department, Hospital Italiano de Buenos Aires, Buenos Aires, Argentina.
}

MaILING AdDREss:

David Aldo De Luca

E-mail: daviddeluca@gmail.com 
dated Dec. 26, 2013) and the informed consent, and we adhered to the ethical principles of the Helsinki Declaration, ethical codes of ANMAT 5330/07 disposition and guidelines for Good Clinical Practice ICH E6. We obtained the informed consent of every patient included in this protocol. The sample size was determined using software GPower V 3.1.7, with a minimum size of 51 patients, distributed in 17 plaque-stage ES-MF patients and 34 healthy patients. Eligible MF patients older than 18 years were included according to the WHO-EORTC criteria. Material and methods are summarized in chart 1. 2,7
We recruited 17 patients with plaque-stage ES-MF and 34 normal skin samples from non-skin cancer patients. Mean age of MF patients was 65.4 years (+/- 28 years), a sex ratio male: female of 14:3 and the disease staging during protocol was $n=3$ (IA) and $\mathrm{n}=14$ (IB). DNA amplification for c-kit gene exons 9, 11, 13, 17 by PCR was performed in all samples (34 healthy skin, 17 plaque-stage ES-MF, and 17 perilesional skin). DNA amplification in exon 9 was not achieved in patient 12, even though PCR conditions were modified. The rest of the samples showed $100 \%$ nucleotide homology, compared to NCBI reference sequence. Regarding CD117 staining,

\section{CHART 1: Material and methods}

Skin samples: Two 4mm punch biopsies: one from plaque-MF, one from the apparently healthy perilesional skin. Excess healthy skin samples from non-cancer surgeries.

DNA extraction and purification: QIAamp ${ }^{\circledast}$ DNA Mini Kit 250 (catalog number 51306) in accordance to manufacturer's catalog specifications.

Gene amplification by PCR for exons 9, 11, 13 and 17 of c-kit gene: specific flanking primers and "AB Applied Biosystems 2720 " thermal cycler. ${ }^{7}$

DNA standard sequencing by Sanger method with Applied Biosystems 3730xl DNA Analyzer (Macrogen ${ }^{\circledast}$, South Korea).

DNA sequence alignment with NCBI Reference Sequence Database, employing online Basic Local Alignment Search Tool (Blast, https:/ / blast.ncbi.nlm.nih.gov /) and SeqMan NGen version 12.0 for multiple sequence alignment.

CD117 expression IHC: automated immunostainer Ventana Benchmark XT ${ }^{\circledast}$ and XT ultraview DAB detection kit (Ventana Medical Systems ${ }^{\circledR}$, Tucson, AZ).

IHC samples: 7 plaque-stage ES-MF, 4 tumor-stage MF, one GIST as a positive control.
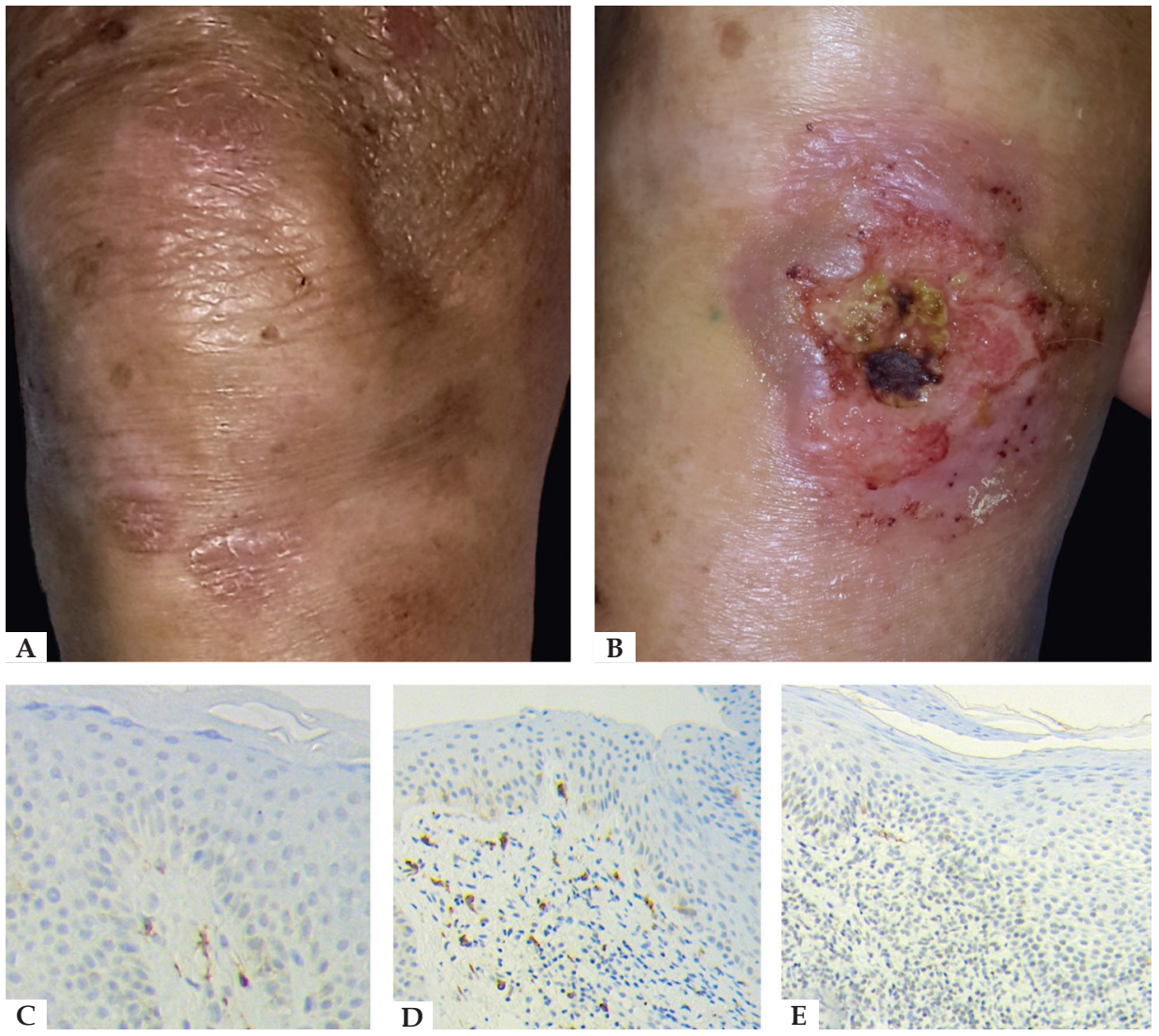

Figure 1: A - Patient 12 with plaque-stage MF on right knee, previous skin biopsies for PCR. B - Nine months later, the same patient developed an ulcerated tumor on right knee. C - CD117 immunohistochemistry: normal skin with low-staining melanocytes and high-staining dermal dendritic cells $\times 20$; D - Plaque stage MF with non-staining atypical T-cells x10; E - Plaque stage MF with low-staining atypical lymphocytes in dermis x10 (Patient 12) 
GIST positive control and all biopsies showed normal CD117+ cells, melanocytes and dendritic cells. Patient 12 showed a low expression of CD117+ in dermal atypical T-cells and tumor-stage MF samples did not express CD117.

c-kit plays a fundamental role during the ontogeny of T-cell, and specific mutations drive to a blockade of pro-T cell development. There is a complex relationship between c-kit and other signal pathways (i.e. NOTCH and IL-7) to induce survival, proliferation, and maturation of T-cells. Regardless of the intricate association, c-kit gene expression is no longer detectable in pre-T-cells and later stages, and the receptor is not necessary for the mature T-cell to survive. As a result of nucleotide variations on regulatory sequences, silent genes like c-kit could be re-expressed in MF T-cells in a monoclonal or oligoclonal fashion. The presence of CD117 in advanced-stage MF and Sézary syndrome could be related to a worse outcome and a lower survival. In our study, CD117 stained with low intensity for atypical T-cells in an 85-year-old type II skin phototype male with ES-MF. It is also remarkable that this patient developed ulcerated tumors months after concluding the study (Figure 1). It is well known that atypical T-cells in MF expand in a polyclonal fashion, and consequently, not every clone would express the same mutations. Furthermore, it is important to remark the fact that Sanger sequencing has a low sensitivity threshold when assessing variant detection status on heterogeneous tumor samples. Sanger method is only capable of detecting mutations when the minor alleles are higher than $20 \%{ }^{8-10}$

As a result of our research, we do not recommend that c-kit gene mutations in exons 9, 11, 13 and 17 should be analyzed by PCR as routine in plaque-stage ES-MF. A whole gene sequencing would provide a better understanding of the phenotype changes and the inconstant re-expression of CD117 in atypical T-cells on advanced-stage MF. $\square$

\section{REFERENCES}

1. Wilcox RA. Cutaneous T-cell lymphoma: 2017 update on diagnosis, riskstratification, and management. Am J Hematol. 2017;92:1085-102.

2. Willemze R, Jaffe ES, Burg G, Cerroni L, Berti E, Swerdlow SH, et al. WHO-EORTC classification for cutaneous lymphomas. Blood. 2005;105:3768-85.

3. Olsen E, Vonderheid E, Pimpinelli N, Willemze R, Kim Y, Knobler R, et al. Revisions to the staging and classification of mycosis fungoides and Sezary syndrome: a proposal of the International Society for Cutaneous Lymphomas (ISCL) and the cutaneous lymphoma task force of the European Organization of Research and Treatment of Cancer (EORTC). Blood. 2007;110:1713-22

4. Stankov K, Popovic S, Mikov M. C-KIT signaling in cancer treatment. Curr Pharm Des. 2014;20:2849-80.

5. Brauns TC, Schultewolter T, Dissemond J, Maschke J, Goos M. C-KIT expression in primary cutaneous T-cell lymphomas. J Cutan Pathol. 2004;31:577-82.

6. Escribano L, Ocqueteau M, Almeida J, Orfao A, San Miguel JF. Expression of the c-kit (CD117) molecule in normal and malignant hematopoiesis. Leuk Lymphoma. 1998;30:459-66
7. Palmirotta R, De Marchis ML, Ludovici G, Leone B, Covello R, Conti S, et al. Mutational analysis of gastrointestinal stromal tumors (GISTs): procedural approach for diagnostic purposes. Cancer Genomics Proteomics. 2013;10:115-23.

8. Xu C, Wan C, Wang L, Yang HJ, Tang Y, Liu WP. Diagnostic significance of TCR gene clonal rearrangement analysis in early mycosis fungoides. Chin J Cancer. 2011;30:264-72.

9. Massa S, Balciunaite G, Ceredig R, Rolink AG. Critical role for c-kit (CD117) in $\mathrm{T}$ cell lineage commitment and early thymocyte development in vitro. Eur $\mathrm{J}$ Immunol. 2006;36:526-32.

10. Davidson CJ, Zeringer E, Champion KJ, Gauthier MP, Wang F, Boonyaratanakornkit $\mathrm{J}$, et al. Improving the limit of detection for Sanger sequencing: A comparison of methodologies for KRAS variant detection. Biotechniques. 2012;53:182-18.

\section{AUTHORS'CONTRIBUTIONS}

David Aldo De Luca $\quad$ (iD) ORCID 0000-0002-2107-301X

Approval of the final version of the manuscript, Conception and planning of the study, Elaboration and writing of the manuscript, Obtaining, analyzing and interpreting the data, Effective participation in research orientation, Intellectual participation in propaedeutic and/or therapeutic conduct of the cases studied, Critical review of the literature

Paula Andrea Enz $\quad$ (iD) ORCID 0000-0002-0890-1642

Approval of the final version of the manuscript, Critical review of the literature, Critical review of the manuscript

Ricardo Luis Galimberti $\quad$ (iD) ORCID 0000-0002-0117-3558

Approval of the final version of the manuscript, Conception and planning of the study, Intellectual participation in propaedeutic and/or therapeutic conduct of the cases studied, Critical review of the literature, Critical review of the manuscript

Adriana Raquel Rinflerch $\quad$ (iD) ORCID 0000-0002-1840-9993

Statistical analysis, Approval of the final version of the manuscript, Conception and planning of the study, Obtaining, analyzing and interpreting the data, Effective participation in research orientation, Intellectual participation in propaedeutic and/or therapeutic conduct of the cases studied, Critical review of the literature, Critical review of the manuscript

How to cite this article: De Luca DA, Enz PA, Galimberti RL, Rinflerch AR. No detection of c-kit gene mutations in exons 9, 11, 13 and 17 and low CD117 expression in plaque-stage mycosis fungoides. An Bras Dermatol. 2018;93(6):913-5. 